\title{
A new thermo-elasto-plasticity constitutive equation for crystals ${ }^{\dagger}$
}

\author{
CHEN Cen, TANG QiHeng \& WANG TzuChiang* \\ State Key Laboratory of Nonlinear Mechanics, Institute of Mechanics, Chinese Academy of Sciences, Beijing 100190, China
}

Received November 6, 2014; accepted December 29, 2014; published online January 20, 2015

\begin{abstract}
Based on the crystal plasticity theory and interatomic potential, in this paper a new thermo-elasto-plasticity constitutive model is proposed to study the behavior of metal crystals at finite temperature. By applying the present constitutive model, the stress-strain curves under uniaxial tension at different temperatures are calculated for the typical crystal Al, and the calculated results are compared with the experimental results. From the comparisons, it can be seen that the present theory has the capability to describe the thermo-elasto-plastic behavior of metal crystals at finite temperature through a concise and explicit calculation process.
\end{abstract}

thermo-elasto-plasticity theory, constitutive equation, interatomic potential, critical resolved shear stress, hardening

PACS number(s): 83.60.La, 91.60.Dc, 81.40.Ef

Citation: Chen C, Tang Q H, Wang T C. A new thermo-elasto-plasticity constitutive equation for crystals. Sci China-Phys Mech Astron, 2015, 58: 054602, doi: $10.1007 / \mathrm{s} 11433-015-5642-2$

The behavior of metal crystals at different temperatures has always extensively drawn attention of scholars. Previously, a series of experiments were carried out to obtain the stressstrain relations of crystals with temperature effect [1-6]. From the experimental results, it can be seen that the stressstrain curves of metal crystals usually diverge with increasing strains at different temperatures. In addition, the divergence is not only evident at high temperatures where thermal softening is rapid, it also appears at low temperatures where softening is usually considered to be ignored.

In recent years, many theoretical investigations have been carried out [7-11]. For example, the molecular dynamics (MD) and Monte Carlo (MC) methods have been quickly developed for the study of temperature effect at the atomistic scale. However, they are not suitable for large scale simulations, and the free energy is difficult to determine [7]. Therefore, several multi-scale modeling methods which combine atomic interaction with continuum models have made significant contributions [9-11], such as the

*Corresponding authors (email: tcwang@imech.ac.cn)

$\uparrow$ Recommended by ZHAO YaPu (Associate Editor) quasicontinuum Monte Carlo (QCMC) method [12] and QC free energy minimization (QCFEM) method [13]. The QCMC method and QCFEM method are the extensions of the quasicontinuum (QC) method [14,15], which is only applicable to the absolute zero temperature, because the interatomic potential does not account for the temperature effect.

Although these theoretical investigations are mature for the thermo-elastic calculation, it is clear that they could do nothing for some experimental results of crystals [1-6]. In these experiments, the plastic behavior plays an important role in the deformation process, and the critical resolved shear stress and hardening are reduced with the temperature increment. Some physical and phenomenological models based on the plastic behavior of metal crystals have been developed [16-20]. Based on the crystal plasticity, the physical models focus on the deformation mechanisms, and have made significant progress. For example, Beyerlein et al. [19] developed a dislocation-based thermally activated hardening model to predict the plastic anisotropy of pure $\mathrm{Zr}$ at different temperatures and under different orientation conditions. The model proposed by Stainier et al. [20] could capture the dependence of the initial yield stress and hardening on temper- 
ature. Moreover, additional forthright models were proposed with easy computer simulation process, such as the JohnsonCook model [21], Zerilli-Armstrong model [22] and KhanHuang-Liang model [16-18]. These models improved the constitutive descriptions of the dynamic plasticity of metals and described the strain, strain- rate, and temperature relations for metals in the large strain, high strain-rate regime. Among these models, the Khan- Huang-Liang model was well developed with a new relation to describe the dependence of work-hardening behavior on the strain and strain rates. It predicted stress-strain response of FCC metals [23], BCC metals [17,24], HCP metals [25], and other metals $[26,27]$, with a good agreement between the simulations and experimental results at different temperatures.

On the other hand, in addition to the widely recognized theories mentioned above, some new temperature-dependent elasto-plastic constitutive models which combine microand macro-scales have been proposed for some complex and difficult problems [28-33]. For example, the crystal plasticity finite-element (CPFE) models have been used to investigate the effect of the dislocation creep [34], hardening behavior [35], and crystal orientation [36] on the plastic behavior of metal at different temperatures. Zbib and Diaz de la Rubia [30] established a hybrid model to investigate size-dependent small-scale plasticity phenomena and related material instabilities at various length scales, which provides an explicit and effective method for the complex plasticity problem. The model proposed by Kim et al. [32] described the cyclic plasticity deformation of $\mathrm{Mg}$ alloys at both the micro- and macro-scales accurately, and had the capacity to deal with complex loading path changes. These multiscale models have addressed problems from new perspectives and made important contributions to the investigation of temperature effect on material behavior.

In the present paper, a new thermo-elasto-plasticity constitutive equation is proposed based on the interatomic potential and solid mechanics. The new deformation decompositions are given in sect. 1.1, in which the total deformation contains thermal, elastic and plastic parts. The thermal strain is introduced in sect. 1.2. The increment constitutive equations are established at the different deformation stages in sect. 1.3. Then the temperature dependences of initial critical resolved shear stress and hardening modulus are considered in sect. 2. The stress-strain curves of $\mathrm{Al}$ crystals at different temperatures are calculated using the present model, and the calculation results are compared with experiments in sect. 3 .

\section{Thermo-elasto-plasticity constitutive rela- tionship}

\subsection{Decomposition of the deformation gradient}

As a crystal material is subjected to the external load at the finite temperature, its deformation should include both the mechanical deformation due to the external load and thermal deformation due to temperature. Therefore, the total deformation of a crystal should be a superposition of the elastic-plastic deformation and the thermal deformation due to the thermal vibration of the atoms around the crystal lattice.

The kinematical theory for the mechanics of elastic- plastic deformation of crystal has been well established [37]. Asaro [37] pointed out that the plastic deformation was due to slip and the elastic deformation was caused by distortion and rotation. The kinematical theory would encounter difficulty when it reaches thermal deformation at a finite temperature, thus a new decomposition of deformation gradient is proposed to describe the thermo-elasto-plasticity deformation behaviour. As shown in Figure 1, the deformation process is decomposed into four parts: the initial configuration at the undeformed state of $0 \mathrm{~K}$ (Figure 1(a)); the first intermediate configuration after free thermal expansions at $T$ K (Figure 1(b)); the second intermediate configuration after elastic deformation at $T \mathrm{~K}$ (Figure 1(c)); and the current configuration after plastic deformation at $T \mathrm{~K}$ (Figure $1(d))$.

The total deformation gradient is decomposed as follows:

$$
\boldsymbol{F}=\boldsymbol{F}^{\mathrm{p}} \boldsymbol{F}^{\mathrm{e}} \boldsymbol{F}^{*},
$$

where $\boldsymbol{F}^{\mathrm{e}}$ is the elastic deformation gradient, $\boldsymbol{F}^{\mathrm{p}}$ is the plastic deformation gradient, and $\boldsymbol{F}^{*}$ is the thermal deformation gradient due to the free thermal expansion.

The thermal strain tensor $\boldsymbol{E}^{*}$, elastic strain tensor $\boldsymbol{E}^{\mathrm{e}}$ and plastic strain tensor $\boldsymbol{E}^{\mathrm{p}}$ take the following respective forms:

$$
\begin{aligned}
& \boldsymbol{E}^{*}=\frac{1}{2}\left(\boldsymbol{F}^{* \mathrm{~T}} \boldsymbol{F}^{*}-\boldsymbol{I}\right), \\
& \boldsymbol{E}^{\mathrm{e}}=\frac{1}{2}\left(\boldsymbol{F}^{\mathrm{eT}} \boldsymbol{F}^{\mathrm{e}}-\boldsymbol{I}\right),
\end{aligned}
$$

(d) Current configuration $T \mathrm{~K}$

(c) Second intermediate

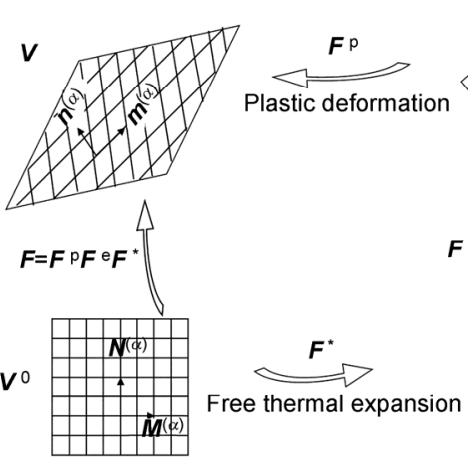

(a) Initial configuration $0 \mathrm{~K}$
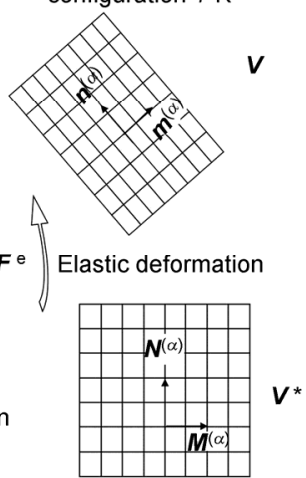

(b) First intermediate configuration $T \mathrm{~K}$

Figure 1 Decomposition of deformation configuration: (a) initial configuration; (b) first intermediate configuration; (c) second intermediate configuration; (d) current configuration. 


$$
\boldsymbol{E}^{\mathrm{p}}=\frac{1}{2}\left(\boldsymbol{F}^{\mathrm{pT}} \boldsymbol{F}^{\mathrm{p}}-\boldsymbol{I}\right)
$$

Therefore, the total strain tensor is expressed as follows:

$$
\begin{aligned}
\boldsymbol{E} & =\frac{1}{2}\left(\boldsymbol{F}^{\mathrm{T}} \boldsymbol{F}-\boldsymbol{I}\right) \\
& =\frac{1}{2}\left[\left(\boldsymbol{F}^{* \mathrm{~T}} \boldsymbol{F}^{\mathrm{eT}} \boldsymbol{F}^{\mathrm{pT}} \boldsymbol{F}^{\mathrm{p}} \boldsymbol{F}^{\mathrm{e}} \boldsymbol{F}^{*}\right)-\boldsymbol{I}\right] \\
& =\frac{1}{2}\left\{\left[\boldsymbol{F}^{* \mathrm{~T}} \boldsymbol{F}^{\mathrm{eT}}\left(2 \boldsymbol{F}^{\mathrm{p}}+\boldsymbol{I}\right) \boldsymbol{F}^{\mathrm{e}} \boldsymbol{F}^{*}\right]-\boldsymbol{I}\right\} \\
& =\frac{1}{2}\left(\boldsymbol{F}^{* \mathrm{~T}} \boldsymbol{F}^{\mathrm{eT}} \boldsymbol{F}^{\mathrm{e}} \boldsymbol{F}^{*}-\boldsymbol{I}\right)+\boldsymbol{F}^{* \mathrm{~T}} \boldsymbol{F}^{\mathrm{eT}} \boldsymbol{E}^{\mathrm{p}} \boldsymbol{F}^{\mathrm{e}} \boldsymbol{F}^{*} \\
& =\boldsymbol{E}^{*}+\boldsymbol{F}^{* \mathrm{~T}} \boldsymbol{E}^{\mathrm{e}} \boldsymbol{F}^{*}+\boldsymbol{F}^{* \mathrm{~T}} \boldsymbol{F}^{\mathrm{eT}} \boldsymbol{E}^{\mathrm{p}} \boldsymbol{F}^{\mathrm{e}} \boldsymbol{F}^{*} .
\end{aligned}
$$

Based on the polar decomposition of the tensor, the deformation gradients $\boldsymbol{F}^{*}$ and $\boldsymbol{F}^{\mathrm{e}}$ are written respectively as:

$$
\begin{aligned}
& \boldsymbol{F}^{*}=\boldsymbol{R}^{*} \boldsymbol{U}^{*}, \\
& \boldsymbol{F}^{\mathrm{e}}=\boldsymbol{R}^{\mathrm{e}} \boldsymbol{U}^{\mathrm{e}},
\end{aligned}
$$

where $\boldsymbol{R}^{*}$ and $\boldsymbol{R}^{\mathrm{e}}$ are the rotation tensors, and $\boldsymbol{U}^{*}$ and $\boldsymbol{U}^{\mathrm{e}}$ are the stretch tensors.

Assuming that $\boldsymbol{R}^{*}=\boldsymbol{I}, \boldsymbol{R}^{\mathrm{e}}=\boldsymbol{I}$, the total strain tensor is expressed as:

$$
\boldsymbol{E}=\boldsymbol{E}^{*}+\boldsymbol{U}^{*} \boldsymbol{E}^{\mathrm{e}} \boldsymbol{U}^{*}+\boldsymbol{U}^{*} \boldsymbol{U}^{\mathrm{e}} \boldsymbol{E}^{\mathrm{p}} \boldsymbol{U}^{\mathrm{e}} \boldsymbol{U}^{*}
$$

Based on eqs. (2a), (2b), (4) and (5), we can obtain the following:

$$
\begin{aligned}
& \boldsymbol{E}^{*}=\frac{1}{2}\left[\left(\boldsymbol{U}^{*}\right)^{2}-\boldsymbol{I}\right], \\
& \boldsymbol{E}^{\mathrm{e}}=\frac{1}{2}\left[\left(\boldsymbol{U}^{\mathrm{e}}\right)^{2}-\boldsymbol{I}\right] .
\end{aligned}
$$

The Taylor expansions of $\boldsymbol{U}^{*}$ and $\boldsymbol{U}^{\mathrm{e}}$ are

$$
\begin{aligned}
& \boldsymbol{U}^{*}=\left(\boldsymbol{I}+\mathbf{2} \boldsymbol{E}^{*}\right)^{1 / 2}=\boldsymbol{I}+\boldsymbol{E}^{*}-\frac{1}{2}\left(\boldsymbol{E}^{*}\right)^{2}+\cdots, \\
& \boldsymbol{U}^{\mathrm{e}}=\left(\boldsymbol{I}+\mathbf{2} \boldsymbol{E}^{\mathrm{e}}\right)^{1 / 2}=\boldsymbol{I}+\boldsymbol{E}^{\mathrm{e}}-\frac{1}{2}\left(\boldsymbol{E}^{\mathrm{e}}\right)^{2}+\cdots,
\end{aligned}
$$

If the thermal strain tensor $\boldsymbol{E}^{*}$ and elastic strain tensor $\boldsymbol{E}^{\mathrm{e}}$ are small, we can obtain

$$
\begin{aligned}
& \boldsymbol{U}^{*} \cong \boldsymbol{I}+\boldsymbol{E}^{*}, \\
& \boldsymbol{U}^{\mathrm{e}} \cong \boldsymbol{I}+\boldsymbol{E}^{\mathrm{e}},
\end{aligned}
$$

and

$$
\begin{aligned}
& \boldsymbol{U}^{*} \cong \boldsymbol{I}, \\
& \boldsymbol{U}^{\mathrm{e}} \cong \boldsymbol{I} .
\end{aligned}
$$

Then, the total strain tensor takes the form:

$$
\boldsymbol{E}=\boldsymbol{E}^{*}+\boldsymbol{E}^{\mathrm{e}}+\boldsymbol{E}^{\mathrm{p}}
$$

Eq. (11) is a new strain tensor expression of the thermal, elastic and plastic deformation at the finite temperature, and it extends the kinematical theory of the elastic-plastic deformation of the crystal [37].

\subsection{Thermal strain}

When an undeformed body is heated up from temperature $T_{0}$ to $T$ and expands freely, the thermal strain is given by [38]

$$
\varepsilon_{T}=\int_{T_{0}}^{T} \alpha \mathrm{d} T
$$

where $T_{0}$ is the reference temperature and is chosen to be room temperature in the present work, $\varepsilon_{T}$ is the thermal strain from temperature $T_{0}$ to temperature $T$, and $\alpha$ is the coefficient of thermal expansion. The coefficient of thermal expansion $\alpha$ can be obtained from the experimental results [39], and can also be calculated by the theoretical method [28,29].

For the metal crystal Al, the thermal strain tensor $\boldsymbol{E}^{*}$ is as follows:

$$
\boldsymbol{E}^{*}=\left[\begin{array}{ccc}
\varepsilon_{T} & 0 & 0 \\
0 & \varepsilon_{T} & 0 \\
0 & 0 & \varepsilon_{T}
\end{array}\right] .
$$

Then the calculations for lattice constant $r^{(0)}(T)$ at temperature $T$ were given by Jiang et al. [9] as follows:

$$
r^{(0)}(T)=r^{(0)}\left(T_{0}\right)\left(1+\int_{T_{0}}^{T} \alpha \mathrm{d} T\right) .
$$

\subsection{Thermo-elasto-plasticity constitutive equations}

The second Piola-Kirchhoff stress is expressed as:

$$
\boldsymbol{S}=\frac{\partial W}{\partial \boldsymbol{E}^{\mathrm{e}}}=\frac{1}{V^{*}}\left[\frac{\partial U_{\text {tot }}\left(\boldsymbol{E}^{\mathrm{e}}\right)}{\partial \boldsymbol{E}^{\mathrm{e}}}\right],
$$

where $V^{*}$ is the volume at the first intermediate configuration as shown in Figure 1(b), $U_{\text {tot }}$ is the total potential energy of the system.

Defining the first intermediate configuration as the reference configuration, the rate of the second Piola-Kirchhoff stress takes the following form:

$$
\begin{aligned}
\dot{\boldsymbol{S}} & =\frac{1}{V^{*}}\left[\frac{\partial U_{\text {tot }}^{2}\left(\boldsymbol{E}^{\mathrm{e}}\right)}{\partial \boldsymbol{E}^{\mathrm{e}} \partial \boldsymbol{E}^{\mathrm{e}}}\right]: \dot{\boldsymbol{E}}^{\mathrm{e}} \\
& =\frac{1}{V^{*}}\left[\frac{\partial U_{\text {tot }}^{2}\left(\boldsymbol{E}^{\mathrm{e}}\right)}{\partial \boldsymbol{E}^{\mathrm{e}} \partial \boldsymbol{E}^{\mathrm{e}}}\right]:\left(\dot{\boldsymbol{E}}-\dot{\boldsymbol{E}}^{\mathrm{p}}-\dot{\boldsymbol{E}}^{*}\right) .
\end{aligned}
$$

The thermo-elastic-plastic constitutive eq. (16) is estab- 
lished by the rate of the second Piola-Kirchhoff stress and the rate of the Green strain.

The Cauchy stress is written as follows:

$$
\begin{aligned}
\boldsymbol{\sigma} & =\frac{1}{V} \boldsymbol{F}^{\mathrm{e}}\left[\frac{\partial U_{\text {tot }}\left(\boldsymbol{E}^{\mathrm{e}}\right)}{\partial \boldsymbol{E}^{\mathrm{e}}}\right] \boldsymbol{F}^{\mathrm{e} T} \\
& =\frac{V^{*}}{V} \boldsymbol{F}^{\mathrm{e}} \boldsymbol{S} \boldsymbol{F}^{\mathrm{e} T}=\frac{1}{J} \boldsymbol{F}^{\mathrm{e}} \boldsymbol{S} \boldsymbol{F}^{\mathrm{e} T},
\end{aligned}
$$

where $V$ is the volume at the second intermediate configuration as shown in Figure $1(\mathrm{c})$, and $J=\frac{V}{V^{*}}$ is the ratio of volume in the second intermediate configuration to that in the first intermediate configuration.

Then the rate of Cauchy stress takes form:

$$
\begin{aligned}
\dot{\boldsymbol{\sigma}} & =\dot{\boldsymbol{F}}^{\mathrm{e}}\left(\boldsymbol{F}^{\mathrm{e}}\right)^{-1} \boldsymbol{\sigma}+\boldsymbol{\sigma}\left(\boldsymbol{F}^{\mathrm{eT}}\right)^{-1}\left(\dot{\boldsymbol{F}}^{\mathrm{e}}\right)^{\mathrm{T}}+\frac{1}{J} \boldsymbol{F}^{\mathrm{e}} \dot{\boldsymbol{S}} \boldsymbol{F}^{\mathrm{eT}}-\frac{\dot{J}}{J} \boldsymbol{\sigma} \\
& =\boldsymbol{L}^{\mathrm{e}} \boldsymbol{\sigma}+\boldsymbol{\sigma} \boldsymbol{L}^{\mathrm{eT}}+\frac{1}{J} \boldsymbol{F}^{\mathrm{e}} \dot{\boldsymbol{S}} \boldsymbol{F}^{\mathrm{eT}}-\frac{\dot{J}}{J} \boldsymbol{\sigma},
\end{aligned}
$$

where $\boldsymbol{L}^{\mathrm{e}}=\dot{\boldsymbol{F}}^{\mathrm{e}}\left(\boldsymbol{F}^{\mathrm{e}}\right)^{-1}$ is the elastic part of the velocity gradient.

The Kirchhoff stress is expressed as follows:

$$
\boldsymbol{\tau}=J \boldsymbol{\sigma}=\boldsymbol{F}^{\mathrm{e}} \boldsymbol{S} \boldsymbol{F}^{\mathrm{eT}} .
$$

Then the rate of Kirchhoff stress takes the following form:

$$
\begin{aligned}
\dot{\boldsymbol{\tau}} & =\dot{\boldsymbol{F}}^{\mathrm{e}} \boldsymbol{S} \boldsymbol{F}^{\mathrm{eT}}+\boldsymbol{F}^{\mathrm{e}} \dot{\boldsymbol{S}} \boldsymbol{F}^{\mathrm{eT}}+\boldsymbol{F}^{\mathrm{e}} \mathbf{S}\left(\dot{\boldsymbol{F}}^{\mathrm{e}}\right)^{\mathrm{T}} \\
& =\dot{\boldsymbol{F}}^{\mathrm{e}}\left(\boldsymbol{F}^{\mathrm{e}}\right)^{-1} \boldsymbol{\tau}+\boldsymbol{\tau}\left(\boldsymbol{F}^{\mathrm{eT}}\right)^{-1}\left(\dot{\boldsymbol{F}}^{\mathrm{e}}\right)^{\mathrm{T}}+\boldsymbol{F}^{\mathrm{e}} \dot{\boldsymbol{S}} \boldsymbol{F}^{\mathrm{e} \mathrm{T}} \\
& =\boldsymbol{L}^{\mathrm{e}} \boldsymbol{\tau}+\boldsymbol{\tau} \boldsymbol{L}^{\mathrm{e} \mathrm{T}}+\boldsymbol{F}^{\mathrm{e}} \dot{\boldsymbol{S}} \boldsymbol{F}^{\mathrm{e} \mathrm{T}} .
\end{aligned}
$$

The Jaumann rate of Kirchhoff stress is

$$
\stackrel{\nabla}{\tau_{\mathrm{I}}}=\dot{\tau}+\boldsymbol{\tau} \boldsymbol{W}^{\mathrm{e}}-\boldsymbol{W}^{\mathrm{e}} \boldsymbol{\tau}
$$

where $\boldsymbol{W}^{\mathrm{e}}$ is the elastic part of the material spin.

Combining eqs. (20) and (21), the following can be obtained:

$$
\begin{aligned}
\boldsymbol{\tau}_{\mathrm{I}}^{\nabla} & =\boldsymbol{F}^{\mathrm{e}} \dot{\boldsymbol{S}} \boldsymbol{F}^{\mathrm{eT}}+\boldsymbol{\tau} \boldsymbol{D}^{\mathrm{e}}+\boldsymbol{D}^{\mathrm{e}} \boldsymbol{\tau} \\
& =\boldsymbol{F}^{e}\left\{\frac{1}{V^{*}}\left[\frac{\partial U_{\mathrm{tot}}^{2}\left(\boldsymbol{E}^{\mathrm{e}}\right)}{\partial \boldsymbol{E}^{\mathrm{e}} \partial \boldsymbol{E}^{\mathrm{e}}}\right]: \dot{\boldsymbol{E}}^{\mathrm{e}}\right\} \boldsymbol{F}^{\mathrm{eT}}+\boldsymbol{\tau} \boldsymbol{D}^{\mathrm{e}}+\boldsymbol{D}^{\mathrm{e}} \boldsymbol{\tau},
\end{aligned}
$$

where $\boldsymbol{D}^{\mathrm{e}}$ is the symmetric rate of the elastic stretching tensor.

The relationship between the elastic part of Green strain rate $\dot{\boldsymbol{E}}^{\mathrm{e}}$ and the symmetric rate of elastic stretching tensor $D^{\mathrm{e}}$ is as follows:

$$
\dot{\boldsymbol{E}}^{\mathrm{e}}=\boldsymbol{F}^{\mathrm{eT}} \boldsymbol{D}^{\mathrm{e}} \boldsymbol{F}^{\mathrm{e}}
$$

Substituting eq. (23) into eq. (22), the following can be obtained:

$$
\begin{aligned}
\tau_{\mathrm{I}}^{\nabla} & =\frac{1}{V^{*}}\left[\left(\boldsymbol{F}^{\mathrm{e}} \otimes \boldsymbol{F}^{\mathrm{e}} \otimes \boldsymbol{F}^{\mathrm{e}} \otimes \boldsymbol{F}^{\mathrm{e}}\right)_{*}^{*} \frac{\partial U_{\mathrm{tot}}^{2}\left(\boldsymbol{E}^{\mathrm{e}}\right)}{\partial \boldsymbol{E}^{\mathrm{e}} \partial \boldsymbol{E}^{\mathrm{e}}}\right] \\
& : \boldsymbol{D}^{\mathrm{e}}+\boldsymbol{D}^{\mathrm{e}} \cdot \boldsymbol{\tau}+\boldsymbol{\tau} \cdot \boldsymbol{D}^{\mathrm{e}} \\
& =\left\{\frac{1}{V^{*}}\left[\left(\boldsymbol{F}^{\mathrm{e}} \otimes \boldsymbol{F}^{\mathrm{e}} \otimes \boldsymbol{F}^{\mathrm{e}} \otimes \boldsymbol{F}^{\mathrm{e}}\right)_{*}^{*} \frac{\partial U_{\mathrm{tot}}^{2}\left(\boldsymbol{E}^{\mathrm{e}}\right)}{\partial \boldsymbol{E}^{\mathrm{e}} \partial \boldsymbol{E}^{\mathrm{e}}}\right]+\overline{\boldsymbol{C}}\right\}: \boldsymbol{D}^{\mathrm{e}} \\
& =\boldsymbol{C}^{\mathrm{e}}: \boldsymbol{D}^{\mathrm{e}},
\end{aligned}
$$

where ${ }_{*}^{*}$ represents quadruple dot products.

And

$$
\boldsymbol{C}^{\mathrm{e}}=\frac{1}{V^{*}}\left[\left(\boldsymbol{F}^{\mathrm{e}} \otimes \boldsymbol{F}^{\mathrm{e}} \otimes \boldsymbol{F}^{\mathrm{e}} \otimes \boldsymbol{F}^{\mathrm{e}}\right)_{*}^{*} \frac{\partial U_{\text {tot }}^{2}\left(\boldsymbol{E}^{\mathrm{e}}\right)}{\partial \boldsymbol{E}^{\mathrm{e}} \partial \boldsymbol{E}^{\mathrm{e}}}\right]+\overline{\boldsymbol{C}} .
$$

The component of $\overline{\boldsymbol{C}}$ is as follows:

$$
\bar{C}_{i j k l}=\tau_{i k} \delta_{j l}+\tau_{k j} \delta_{i l}
$$

For metal crystal, the plastic deformation is determined based on the crystal plasticity theory. As shown in Figure 1, $\boldsymbol{M}^{(\alpha)}$ and $\boldsymbol{N}^{(\alpha)}$ respectively represent the unit vectors in the slip direction and the normal direction to the slip plane for slip system $\alpha$ in the first intermediate configuration. After elastic deformation, the slip direction and the corresponding normal on slip system $\alpha$ become to $\boldsymbol{m}^{(\alpha)}$ and $\boldsymbol{n}^{(\alpha)}$, which satisfy the following:

$$
\begin{gathered}
\boldsymbol{m}^{(\alpha)}=\boldsymbol{F}^{\mathrm{e}} \boldsymbol{M}^{(\alpha)}, \\
\boldsymbol{n}^{(\alpha)}=\left(\left(\boldsymbol{F}^{\mathrm{e}}\right)^{-1}\right)^{\mathrm{T}} \boldsymbol{N}^{(\alpha)} .
\end{gathered}
$$

Defining the second-order symmetric tensor $\boldsymbol{P}^{(\alpha)}$ and the antisymmetric tensor $\boldsymbol{Q}^{(\alpha)}$ as follows:

$$
\begin{aligned}
& \boldsymbol{P}^{(\alpha)}=\frac{1}{2}\left(\boldsymbol{m}^{(\alpha)} \otimes \boldsymbol{n}^{(\alpha)}+\boldsymbol{n}^{(\alpha)} \otimes \boldsymbol{m}^{(\alpha)}\right), \\
& \boldsymbol{Q}^{(\alpha)}=\frac{1}{2}\left(\boldsymbol{m}^{(\alpha)} \otimes \boldsymbol{n}^{(\alpha)}-\boldsymbol{n}^{(\alpha)} \otimes \boldsymbol{m}^{(\alpha)}\right) .
\end{aligned}
$$

The resolved shear stress on the slip system $\alpha$ is

$$
\tau^{(\alpha)}=\boldsymbol{\tau}: \boldsymbol{P}^{(\alpha)} .
$$

The rate of symmetric tensor $\boldsymbol{P}^{(\alpha)}$ is

$$
\dot{\boldsymbol{P}}^{(\alpha)}=\boldsymbol{D}^{\mathrm{e}} \boldsymbol{Q}^{(\alpha)}+\boldsymbol{W}^{\mathrm{e}} \boldsymbol{P}^{(\alpha)}-\boldsymbol{P}^{(\alpha)} \boldsymbol{W}^{\mathrm{e}}-\boldsymbol{Q}^{(\alpha)} \boldsymbol{D}^{\mathrm{e}} .
$$

And the rate of the resolved shear stress on the slip system $\alpha$ is 


$$
\begin{aligned}
\dot{\tau}^{(\alpha)} & =\dot{\boldsymbol{\tau}}: \boldsymbol{P}^{(\alpha)}+\boldsymbol{\tau}: \dot{\boldsymbol{P}}^{(\alpha)} \\
& =\dot{\boldsymbol{\tau}}: \boldsymbol{P}^{(\alpha)}+\left(\boldsymbol{\tau} \boldsymbol{W}^{\mathrm{e}}-\boldsymbol{W}^{\mathrm{e}} \boldsymbol{\tau}\right) \\
& : \boldsymbol{P}^{(\alpha)}+\left(\boldsymbol{Q}^{(\alpha)} \boldsymbol{\tau}-\boldsymbol{\tau} \boldsymbol{Q}^{(\alpha)}\right): \boldsymbol{D}^{\mathrm{e}} \\
& =\boldsymbol{\tau}_{\mathrm{I}}: \boldsymbol{P}^{(\alpha)}+\left(\boldsymbol{Q}^{(\alpha)} \boldsymbol{\tau}-\boldsymbol{\tau} \boldsymbol{Q}^{(\alpha)}\right): \boldsymbol{D}^{\mathrm{e}} \\
& =\left(\boldsymbol{C}^{e}: \boldsymbol{P}^{(\alpha)}+\boldsymbol{Q}^{(\alpha)} \boldsymbol{\tau}-\boldsymbol{\tau} \boldsymbol{Q}^{(\alpha)}\right): \boldsymbol{D}^{\mathrm{e}} \\
& =\boldsymbol{\lambda}^{(\alpha)}: \boldsymbol{D}^{\mathrm{e}},
\end{aligned}
$$

where $\boldsymbol{\lambda}^{(\alpha)}=\boldsymbol{C}^{\mathrm{e}}: \boldsymbol{P}^{(\alpha)}+\boldsymbol{Q}^{(\alpha)} \boldsymbol{\tau}-\tau \boldsymbol{Q}^{(\alpha)}$.

According to the generalized Schmid's law, the resolved shear stress $\tau^{(\alpha)}$ of a potentially active or critical slip system must reach its critical value $\tau_{ \pm c r}^{(\alpha)}$. For a remaining active slip system, $\tau^{(\alpha)}$ must increase to and remain at the critical value, as follows:

$$
\begin{cases}\dot{\tau}_{\mathrm{cr}}^{(\alpha)}=\dot{\tau}^{(\alpha)}=\sum_{\beta=1}^{m} h_{\alpha \beta} \dot{\gamma}^{(\beta)}, & \sum_{\beta=1}^{m} h_{\alpha \beta} \dot{\gamma}^{(\beta)}>0, \\ \dot{\tau}_{-\mathrm{cr}}^{(\alpha)}=\dot{\tau}^{(\alpha)}=\sum_{\beta=1}^{m} h_{\alpha \beta} \dot{\gamma}^{(\beta)}, & \sum_{\beta=1}^{m} h_{\alpha \beta} \dot{\gamma}^{(\beta)}<0,\end{cases}
$$

where $h_{\alpha \beta}$ is the hardening modulus.

Substituting eq. (33) into eq. (34), it can be obtained that for the remaining active slip system the following is true:

$$
\sum_{\beta=1}^{m} h_{\alpha \beta} \dot{\gamma}^{(\beta)}=\lambda^{(\alpha)}: \boldsymbol{D}^{\mathrm{e}} .
$$

In the theory of crystal plasticity, the symmetric and antisymmetric parts of the plastic velocity gradient have the following relationship with the second-order tensors $\boldsymbol{P}^{(\alpha)}$ and $\boldsymbol{Q}^{(\alpha)}$ :

$$
\begin{gathered}
\boldsymbol{L}^{\mathrm{p}}=\sum_{\alpha=1}^{n} \boldsymbol{m}^{(\alpha)} \otimes \boldsymbol{n}^{(\alpha)} \dot{\gamma}^{(\alpha)}, \\
\boldsymbol{D}^{\mathrm{p}}=\sum_{\alpha=1}^{n} \boldsymbol{P}^{(\alpha)} \dot{\gamma}^{(\alpha)} \\
\boldsymbol{W}^{\mathrm{p}}=\sum_{\alpha=1}^{n} \boldsymbol{Q}^{(\alpha)} \dot{\gamma}^{(\alpha)} .
\end{gathered}
$$

The rate of plastic deformation gradient is

$$
\dot{\boldsymbol{F}}^{\mathrm{p}}=\sum_{\alpha=1}^{n} \boldsymbol{M}^{(\alpha)} \otimes \boldsymbol{N}^{(\alpha)} \dot{\gamma}^{(\alpha)} .
$$

The rate of plastic strain can then be obtained based on eq. (39):

$$
\dot{\boldsymbol{E}}^{\mathrm{p}}=\frac{1}{2}\left(\left(\dot{\boldsymbol{F}}^{\mathrm{p}}\right)^{\mathrm{T}} \boldsymbol{F}^{\mathrm{p}}+\boldsymbol{F}^{\mathrm{p} \mathrm{T}} \dot{\boldsymbol{F}}^{\mathrm{p}}\right)
$$

Substituting the rate of plastic strain into the constitutive eq. (16), and combining this with the rate of thermal strain, the increment stress-strain relationship can then be obtained.
Based on eqs. (37) and (35), we may then obtain the following:

$$
\begin{gathered}
\sum_{\beta=1}^{m} h_{\alpha \beta} \dot{\gamma}^{(\beta)}=\boldsymbol{\lambda}^{(\alpha)}:\left(\boldsymbol{D}-\sum_{\alpha=1}^{m} \dot{\gamma}^{(\alpha)} \boldsymbol{P}^{(\alpha)}\right), \\
\dot{\gamma}^{(\alpha)}=\sum_{\beta=1}^{m}\left(g_{\alpha \beta}\right)^{-1} \boldsymbol{\lambda}^{(\beta)}: \boldsymbol{D},
\end{gathered}
$$

where $g_{\alpha \beta}=h_{\alpha \beta}+\lambda^{(\alpha)}: \boldsymbol{P}^{(\beta)}$.

The Jaumann rate of Kirchhoff stress is as follows:

$$
\begin{aligned}
{ }_{\boldsymbol{\tau}}^{\nabla} & =\dot{\boldsymbol{\tau}}+\boldsymbol{\tau} \boldsymbol{W}-\boldsymbol{W} \boldsymbol{\tau} \\
& =\dot{\boldsymbol{\tau}}+\boldsymbol{\tau} \boldsymbol{W}^{\mathrm{e}}-\boldsymbol{W}^{\mathrm{e}} \boldsymbol{\tau}+\boldsymbol{\tau} \boldsymbol{W}^{\mathrm{p}}-\boldsymbol{W}^{\mathrm{p}} \boldsymbol{\tau} .
\end{aligned}
$$

Substituting eqs. (24) and (38) into eq. (43), the following can be obtained:

$$
\begin{aligned}
\nabla & =\boldsymbol{C}^{\mathrm{e}}:\left(\boldsymbol{D}-\boldsymbol{D}^{p}\right)-\sum_{\alpha=1}^{m} \dot{\gamma}^{(\alpha)}\left(\boldsymbol{Q}^{(\alpha)} \boldsymbol{\tau}-\boldsymbol{\tau} \boldsymbol{Q}^{(\alpha)}\right) \\
& =\boldsymbol{C}^{\mathrm{e}}: \boldsymbol{D}-\sum_{\alpha=1}^{m} \dot{\gamma}^{(\alpha)}\left(\boldsymbol{C}^{\mathrm{e}}: \boldsymbol{P}^{(\alpha)}+\boldsymbol{Q}^{(\alpha)} \boldsymbol{\tau}-\boldsymbol{\tau} \boldsymbol{Q}^{(\alpha)}\right) \\
& =\boldsymbol{C}^{\mathrm{e}}: \boldsymbol{D}-\sum_{\alpha=1}^{m} \dot{\gamma}^{(\alpha)} \boldsymbol{\lambda}^{(\alpha)} .
\end{aligned}
$$

Then, substituting eq. (42) into eq. (44), the Jaumann rate of Kirchhoff stress is written as follows:

$$
{ }_{\boldsymbol{\tau}}^{\nabla}=\left(\boldsymbol{C}^{\mathrm{e}}-\sum_{\alpha=1}^{m} \sum_{\beta=1}^{m}\left(g_{\alpha \beta}\right)^{-1} \boldsymbol{\lambda}^{(\beta)} \otimes \boldsymbol{\lambda}^{(\alpha)}\right): \boldsymbol{D},
$$

where $C^{\mathrm{e}}$ is determined by eq. (25). In addition, the constitutive eq. (45) is established by the Jaumann rate of the Kirchhoff stress and the symmetric parts of the velocity gradient in the current configuration.

Generally speaking, crystalline materials possess crystallographic point groups as description of the material symmetries. The microscopic structure of a given material could be unaltered in observation under certain orthogonal transformations. At the same time, the constitutive equations should also remain unchanged when the reference configurations or coordinates transform. And this limit would be reflected by the tensor function of strain and stress.

The constitutive equations in the present model are established by the rate of the second Piola-Kirchhoff stress and the rate of the Green strain (eq. (16)), as well as the Jaumann rate of the Kirchhoff stress and symmetric parts of the velocity gradient (eq. (45)). The stress and strain tensors in the constitutive equations are complete representations; the rate of the second Piola-Kirchhoff stress tensor and the Jaumann rate of the Kirchhoff stress tensor are objective, which would not be affected by the change of crystal's rota- 
tion and change of coordinates. So the constitutive equations in the present model can satisfy the objectivity principle and the requirements of symmetry in the crystal's response.

\section{Temperature effect on the initial critical re- solved shear stress and hardening modulus}

The influence of the temperature on glide has been widely investigated with metal crystals [1-3]. Due to the fact that the initial yield stress and hardening behavior play important roles in the plastic behavior, the determination of the temperature effects on the initial critical resolved shear stress and hardening modulus is necessary for theoretical investigation and practical application.

\subsection{Temperature effect on the initial critical resolved shear stress}

Based on eq. (31), the resolved shear stress on the slip system $\alpha$ under uniaxial loading is written as follows:

$$
\tau^{(\alpha)}=\tau_{11} P_{11}^{(\alpha)} .
$$

For single crystals, the maximal value of $P_{11}^{(\alpha)}$ can be obtained based on the orientation of the slip system. Assuming that the elastic deformation is sufficiently small, the ratio of volume $J=1$. Therefore, the relationship between the critical resolved shear stress and initial yield stress is written as follows:

$$
\tau_{\mathrm{cr}}^{0}=\sigma_{y s}^{0} P_{11}^{\max }
$$

where $P_{11}^{\max }$ is the maximum value of $P_{11}^{(\alpha)}$ among all slip systems.

To obtain the relationship between yield stress and temperature, several representative experimental results are adopted [1-3,40,41], such as the critical resolved shear stress (dot in Figure 2) for BCC crystal $\alpha$-Fe, rhombohedra crystal $\mathrm{Bi}$ and hexagonal crystals $\mathrm{Mg}, \mathrm{Cd}$ and $\mathrm{Zn}$. As shown in Figure 2, when the temperature is below the melting point, the critical shear stress decreases with the rising temperature. By fitting the experimental data with a smooth curve, it can be concluded that the exponential curve is more accurate in describing the relationship between the critical shear stress and temperature. Thus the critical resolved shear stress can be written as follows:

$$
\tau_{\text {cr }}^{0}(T)=A_{1}+B_{1} \mathrm{e}^{-d_{1} T / T_{0}}
$$

where $A_{1}, B_{1}$ and $d_{1}$ are parameters, and $T_{0}$ is room temperature $(293 \mathrm{~K})$.

Similar to the expression of critical resolved shear stress shown in eq. (48), the exponential relationship between the
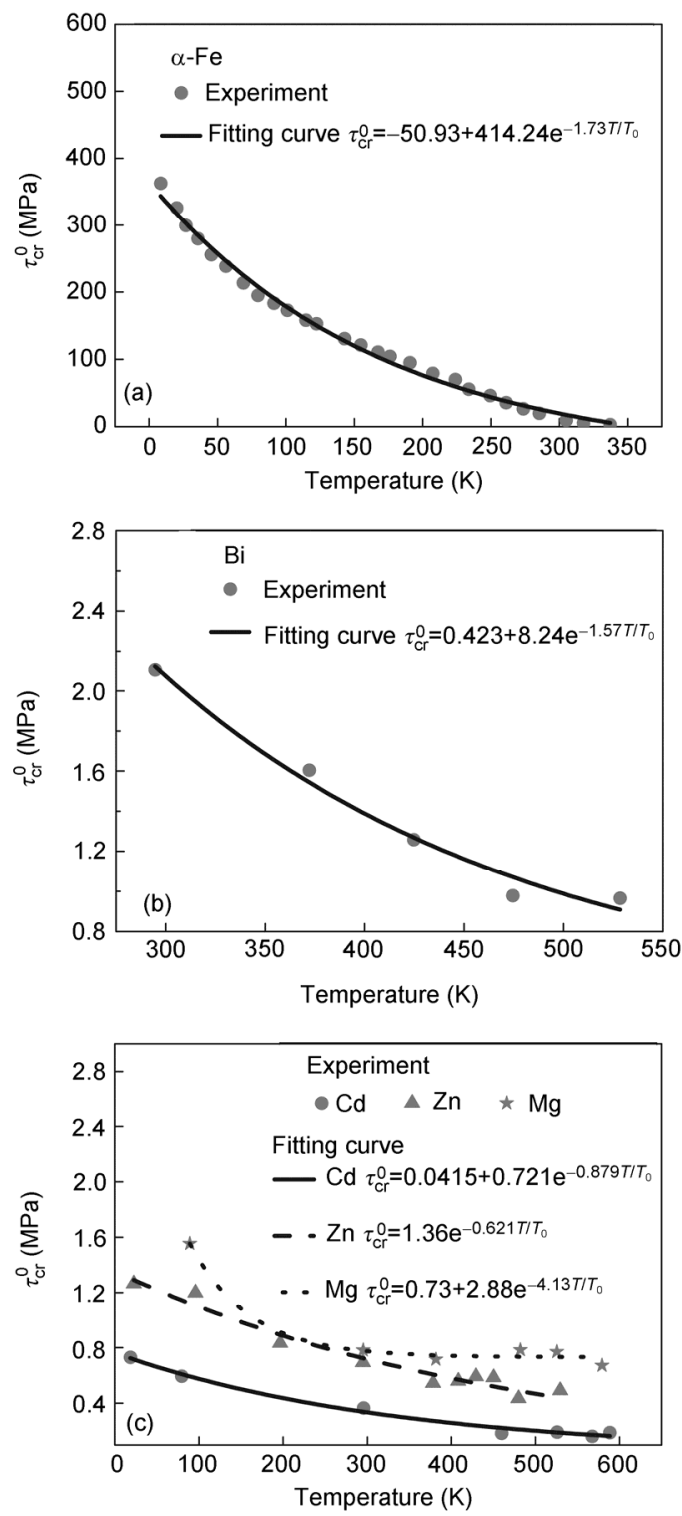

Figure 2 Temperature effect on the critical shear stress for different metal crystals. (a) $\alpha$ - Fe, (b) $\mathrm{Bi}$, (c) $\mathrm{Mg}, \mathrm{Zn}, \mathrm{Cd}$.

critical resolved shear stress and temperature has also been proposed by some researchers [42-45]. Moreover, in Howe's experiment regarding $\mathrm{Al}$ [46], the variation of the yield point with temperature follows a similar rule at the static strain rate.

\subsection{Temperature effect on hardening modulus}

Similar to the exponential law of the macroscopic strainstress curve, which has been effectively adopted by the theoretical model [16,21], the hardening modulus $h_{\alpha \beta}$ is written as follows:

$$
\left\{\begin{array}{l}
h_{\alpha \alpha}=c(T)\left(\gamma^{(\alpha)}+\gamma_{0}(T)\right)^{m-1}, \\
h_{\alpha \beta}=q h_{\alpha \alpha}, \beta \neq \alpha,
\end{array}\right.
$$


where $c(T)$ and $m$ are the kinematic hardening parameters, $q$ is the latent hardening parameter, and $\gamma_{0}(T)=$ $\left(\frac{m \cdot \tau_{\mathrm{cr}}^{0}(T)}{c(T)}\right)^{\frac{1}{m}}$. In addition, $c(T)$ is the function of temperature $T$, and parameter $m$ remains unchanged at different temperatures. Due to the fact that the latent hardening behavior is not accounted for in this paper, the value of latent hardening parameter $q$ is 0 .

Due to the fact that a large number of experiments show that hardening is greatly reduced as the temperature rises [2,3,46-49], we adopt an exponential curve to describe the relationship between the hardening and temperature, and $c(T)$ is calculated as follows:

$$
c(T)=A_{2}+B_{2} \mathrm{e}^{-d_{2} T / T_{0}},
$$

where $A_{2}, B_{2}$ and $d_{2}$ are parameters, and $T_{0}$ is room temperature $(293 \mathrm{~K})$.

\section{Calculation and discussion}

The stress-strain curves of single crystal $\mathrm{Al}$ are calculated at different temperatures, and the calculated results are compared with those of the experiment performed by Boas and Schmid [2]. In their experiment, the Al specimens were strained at different temperatures, and the stress-strain curves were obtained. Since the melting temperature is about $993 \mathrm{~K}$ for $\mathrm{Al}$, we define $0 \mathrm{~K}$ to $500 \mathrm{~K}$ as low temperature, and $500 \mathrm{~K}$ to $993 \mathrm{~K}$ as high temperature.

\subsection{Calculation procedures}

In each calculation step, the elastic strain is given. The calculation procedures are shown in Figure 3 and more details are presented as follows:

(1) The thermal strain and lattice constants at different temperatures are respectively calculated based on eqs. (12) and (14), and the thermal expansion $\alpha$ in eqs. (12) and (14) is obtained from the experimental results [39]. Figures 4 and 5 show the thermal strain and lattice constant versus temperature for Al. Room temperature $(293 \mathrm{~K})$ is chosen as the reference temperature, and the thermal strain at room temperature is 0 .

(2) The increment of the second Piola-Kirchhoff stress is calculated based on eq. (16) with a given increment of elastic strain. The EAM potential proposed by Mei and Davenport [50] is adopted to calculate the potential energy and thermal stress (eq. (16)) for the Al, and the change of the lattice constant with temperature is considered in the calculation of potential energy.

(3) The increment of plastic strain is calculated using eq. (40), and the stress-strain curve is obtained by accumulating

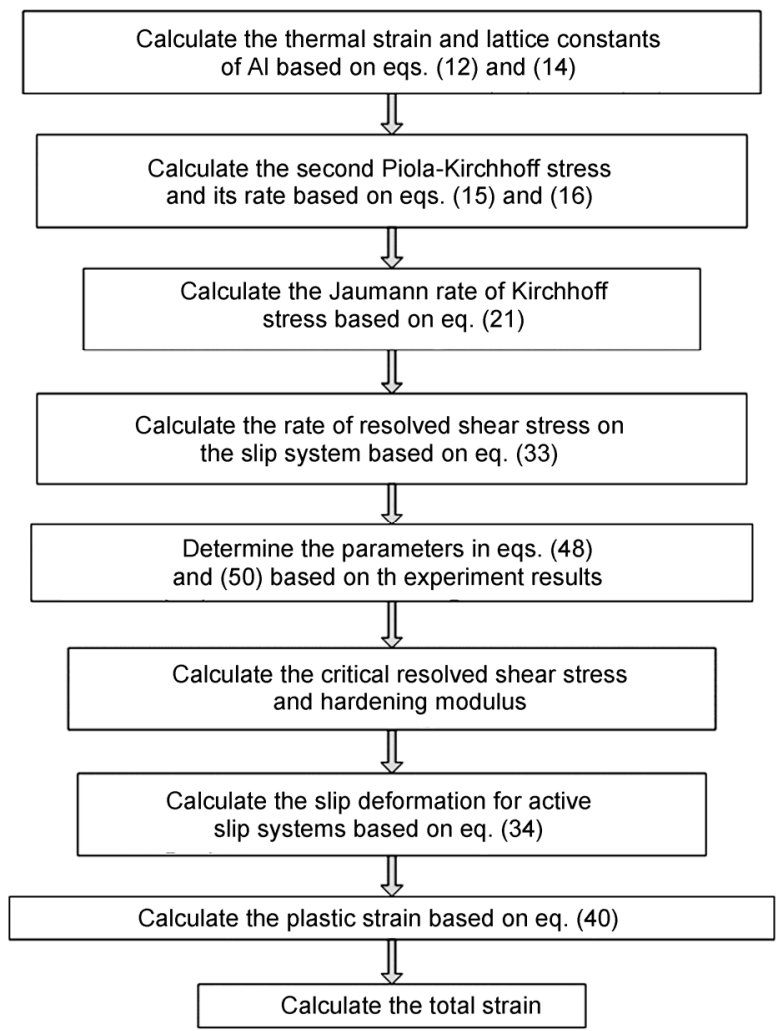

Figure 3 Flow chat of the calculation procedures.

the increment of the stress and strain at each load step.

The calculated parameters are shown in Table 1, which can fluctuate within a deviation range from $5 \%$ to $10 \%$. This deviation would not influence the accuracy of the calculated results. So the present model is insensitive to parameters, which ensures the ability of the present model to describe the material behavior at different temperatures accurately.

The fitting curves of the initial critical resolved shear stress $\tau_{\mathrm{cr}}^{0}$ for $\mathrm{Al}$ are shown in Figure 6. Moreover, the fitting curves of kinematic hardening parameter $c(T)$ are also shown in Figure 7. From several previous investigations, it has been found that above $550 \mathrm{~K}$ the yield point drops rapidly with temperature [46], and the low-temperature and high-temperature work hardening differs in terms of type and intensity [4]. We choose two sets of parameters to respectively describe the changes of the initial critical resolved shear stress and hardening modulus at low- temperature and high- temperature.

It is difficult to obtain $c(T)$ directly from experiments. After parameter $m$ has been determined, the value of $c(T)$ can be obtained by fitting the experimental results given by Boas and Schmid [2]. Finally, the experimental values of $c(T)$ at different temperatures are obtained.

\subsection{Results and discussion}

Figure 8 is the comparisons of the true stress-strain curve 


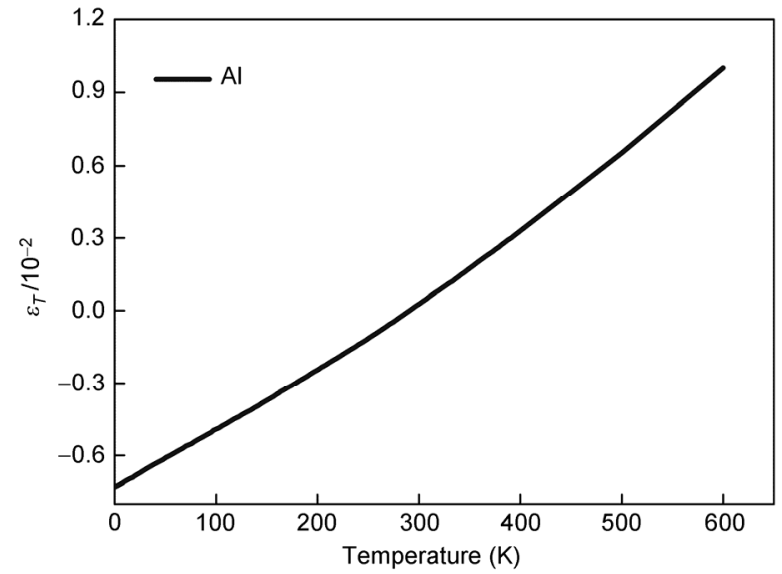

Figure 4 Thermal strain versus temperature for Al.

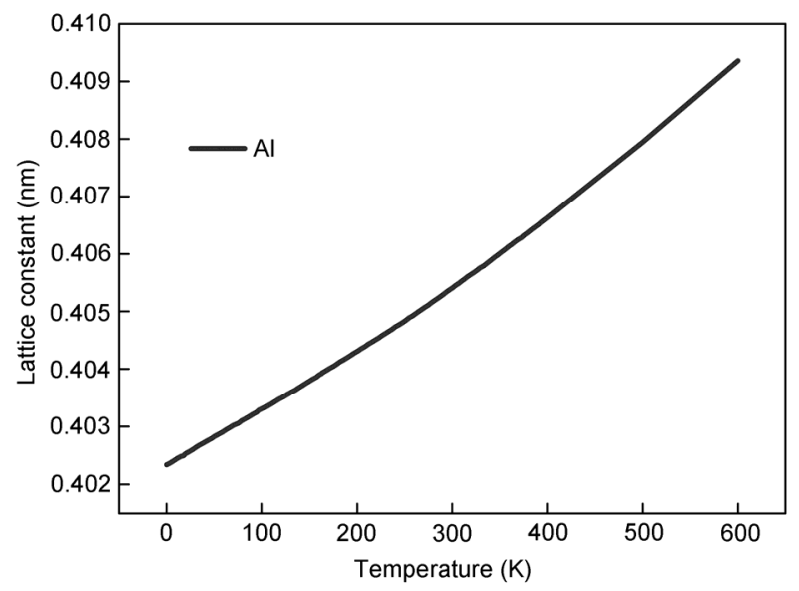

Figure 5 Lattice constant versus temperature for Al.

Table 1 Calculated parameters for Al

\begin{tabular}{cccccccc}
\hline$T(\mathrm{~K})$ & $A_{1}(\mathrm{MPa})$ & $B_{1}(\mathrm{MPa})$ & $d_{1}$ & $A_{2}(\mathrm{MPa})$ & $B_{2}(\mathrm{MPa})$ & $d_{2}$ & $m$ \\
\hline $88-473$ & 3.99 & 5.04 & 1.20 & 29.06 & 168.07 & 1.34 & 0.67 \\
$573-773$ & 0.0796 & 149.55 & 1.86 & 7.6 & 46023 & 3.93 & 0.67 \\
\hline
\end{tabular}

for $\mathrm{Al}$ under uniaxial load between the calculated and experimental results at different temperatures. Figure 8 (a) shows the comparisons for $88 \mathrm{~K}, 291 \mathrm{~K}, 373 \mathrm{~K}$ and $473 \mathrm{~K}$, and Figure 8 (b) shows those for $573 \mathrm{~K}, 673 \mathrm{~K}$ and $773 \mathrm{~K}$.

The results verify that our proposed theory can predict the thermo-elasto-plasticity behavior of metal crystal very effectively. The yield strength and work hardening of crystal decrease with increasing temperature, which has been previously observed by some experiments $[1,3,47]$, and some investigations suggested the thermally activated collapse of sessile dislocations during plastic flow to explain the temperature effect on the work hardening [4,51] and yield strength [52]. Similar to the other theories proposed by some researchers [42-45], the exponential relationships are applied in eqs. (48) and (50).

A new decomposition of the total deformation gradient is proposed in this article, which is different from the kinematical theory described by Asaro [37], who suggested that the total deformation gradient is

$$
\boldsymbol{F}=\boldsymbol{F}^{\mathrm{e}} \boldsymbol{F}^{\mathrm{p}} .
$$

Eq. (51) signifies that the material undergoes plastic slip through the undeformed crystal lattice according to $\boldsymbol{F}^{\mathrm{p}}$ reaching the intermediate configuration, then the material deforms according to $\boldsymbol{F}^{\mathrm{e}}$ reaching the current configuration. The decomposition of the deformation gradient by eq. (51) has been applied extensively by many researchers and made a great contribution to the theoretical advancement of crystal plasticity [53,54].

However, eq. (51) is not effective when it is applied to the thermal strain, meaning that when the total deformation gradient is written as $\boldsymbol{F}=\boldsymbol{F}^{\mathrm{e}} \boldsymbol{F}^{\mathrm{p}} \boldsymbol{F}^{*}$, one may not obtain the simple strain tensor equation as eq. (11). Therefore, it is necessary to adopt the new composition of deformation gradient as eq. (1). The physical phenomenon is objective, and one can use different theories to analyze it. In addition, although both decompositions of deformation gradients $\boldsymbol{F}=\boldsymbol{F}^{\mathrm{p}} \boldsymbol{F}^{\mathrm{e}} \boldsymbol{F}^{*}$, and $\boldsymbol{F}=\boldsymbol{F}^{\mathrm{e}} \boldsymbol{F}^{\mathrm{p}} \boldsymbol{F}^{*}$ may be acceptable, the decompositions $\boldsymbol{F}=\boldsymbol{F}^{\mathrm{p}} \boldsymbol{F}^{\mathrm{e}} \boldsymbol{F}^{*}$, are better than $\boldsymbol{F}=\boldsymbol{F}^{\mathrm{e}} \boldsymbol{F}^{\mathrm{p}} \boldsymbol{F}^{*}$, as a simple and brief mathematical expression is usually favorable.

So the advantages of new decomposition in the present model mostly embody two aspects: the first one is that the thermal deformation is considered in the whole deformation process; the second one is that it provides a good basis to
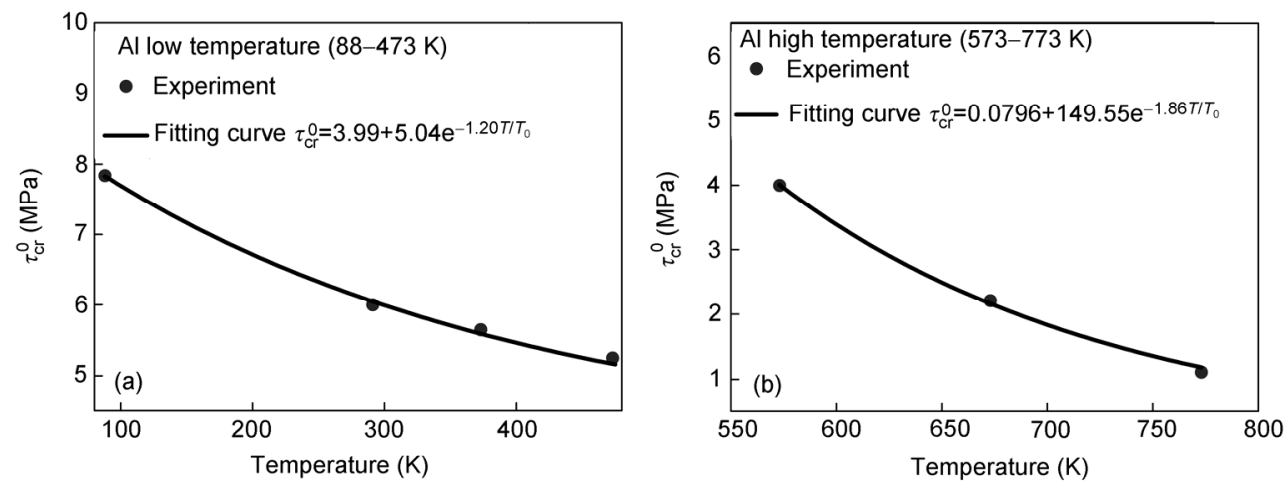

Figure 6 Fitting curves of initial critical resolved shear stress $\tau_{\mathrm{cr}}^{0}$. (a) Low temperature, (b) high temperature. 

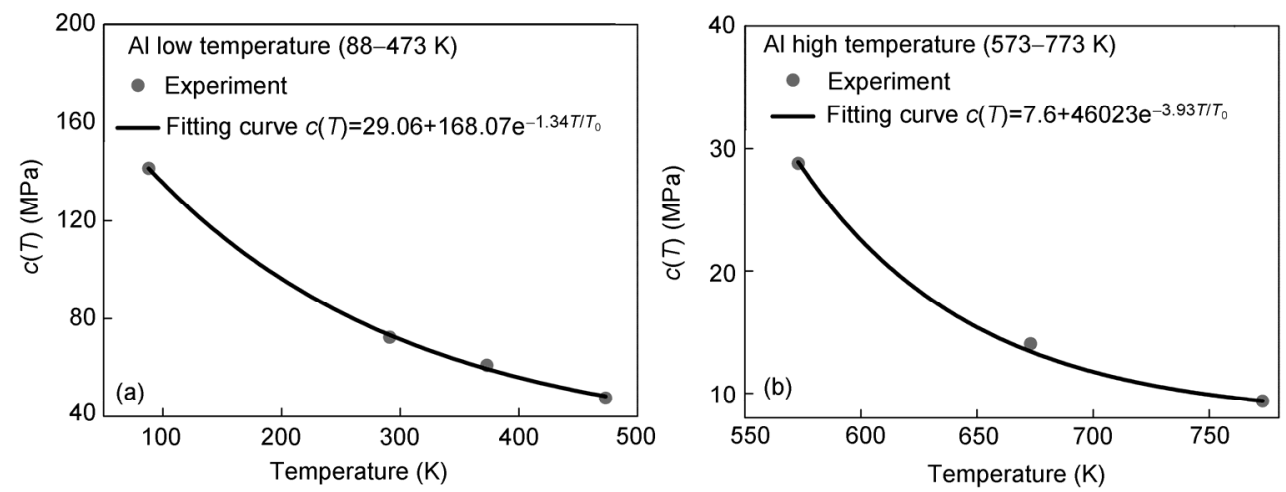

Figure 7 Fitting curves of kinematic hardening parameter $c(T)$. (a) Low temperature, (b) high temperature.
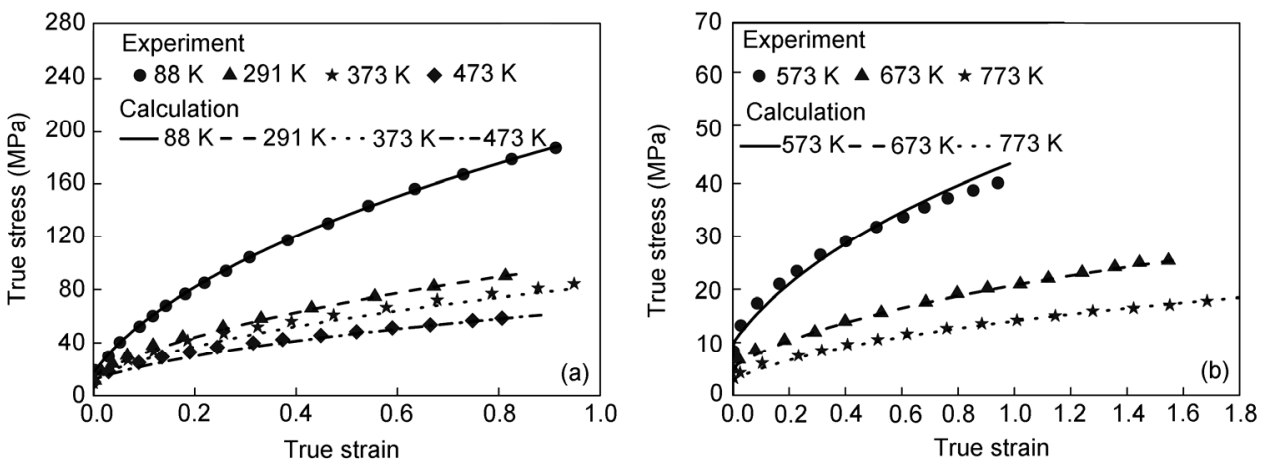

Figure 8 Comparison of true stress-strain curve for $\mathrm{Al}$ between the calculated and experimental results at different temperatures. (a) Low temperature, (b) high temperature.

establish the constitutive equations and makes the calculation process more simple and explicit.

Comparing with some widely recognized models, the present model has some characteristics as follows: firstly, the constitutive equations can be obtained easily based on the new decomposition of deformation gradient. And the computational efficiency is higher than the MD and MC methods. Then, the thermal deformation was not considered in most of the previous models, such as the Johnson-Cook model [21] and the Zerilli-Armstrong model [22]. In the presrnt model, the thermal strain is considered in the whole deformation process, which makes the present model applicable to structural calculation with some boundary constraints in the future. Lastly, the temperature effects on the initial critical resolved shear stress and hardening behaviors are reflected by concise expression, and the parameters can be determined easily with three uniaxial stress-strain curves at different temperature. So the thermo-elasto-plasticity behavior can be described more clearly and accurately.

\section{Conclusions}

In this paper, a new thermo-elasto-plasticity constitutive model is proposed. The stress-strain curves of Al crystal are calculated at different temperatures, and the calculated re- sults are in good agreement with the experiments, which verifies that the present model may effectively predict the elastic and plastic deformation behavior of metal crystal at different temperatures. A new decomposition of total deformation gradient is suggested, and the simple strain tensor equation is obtained based on the new decomposition method.

This work was supported by the National Natural Science Foundation of China (Grant Nos. 11021262, 11172303 and 11132011) and National Basic Research Program of China (Grant No. 2012CB937500).

1 Boas W, Schmid E. Über die Temperaturabhängigkeit der Kristallplastizität. Zeitschrift für Physik, 1930, 61: 767-781

2 Boas W, Schmid E. Über die Temperaturabhängigkeit der Kristallplastizität III Aluminium. Zeitschrift für Physik, 1931, 71: 703-714

3 Schmid E. Beiträge zur Physik und Metallographie des Magnesiums. Zeitschrift für Elektrochemie und angewandte physikalische Chemie, 1931, 37: 447-459

4 Cottrell A, Stokes R. Effects of temperature on the plastic properties of aluminium crystals. Math Phys Sci, 1955, 233: 17-34

5 Hoge K G, Mukherjee A K. The temperature and strain rate dependence of the flow stress of tantalum. J Mater Sci, 1977, 12: 1666-1672

6 Huang S. Direct Disc Impact Investigation of Dynamic Large Finite Plastic Behavior of 1100 Aluminum. Dissertation for Doctoral Degree. Norman: University of Oklahoma, 1990

7 Frenkel D, Ladd A J C. New Monte Carlo method to compute the 
free energy of arbitrary solids. Applicaton to the fcc and hcp phases of hard spheres. J Chem Phys, 1984, 81: 3188-3193

8 Huang Z, Dui G, Yang S. A new interpretation of internal-variable theory in finite thermo-viscoelasticity. Sci China-Phys Mech Astron, 2013, 56: 610-616

9 Jiang H, Huang Y, Hwang K C. A finite-temperature continuum theory based on interatomic potentials. J Eng Mater Technol, 2005, 127: 408-416

10 Li X, E W N. Multiscale modeling of the dynamics of solids at finite temperature. J Mech Phys Solids, 2005, 53: 1650-1685

11 LeSar R, Najafabadi R, Srolovitz D J. Finite-temperature defect properties from free-energy minimization. Phys Rev Lett, 1989, 63: 624-627

12 Miller R E, Tadmor E B. The quasicontinuum method: Overview, applications and current directions. J Comput Aided Mater, 2002, 9: 203-239

13 Dupuy L M, Tadmor E B, Miller R E, et al. Finite-temperature quasicontinuum: Molecular dynamics without all the atoms. Phys Rev Lett, 2005, 95: 060202

14 Miller R, Ortiz M, Phillips R, et al. Quasicontinuum models of fracture and plasticity. Eng Fra Mech, 1998, 61: 427-444

15 Miller R, Tadmor E B, Phillips R, et al. Quasicontinuum simulation of fracture at the atomic scale. Modell. Simul Mater Sci Eng, 1998, 6: 607-638

16 Khan A S, Huang S. Experimental and theoretical study of mechanical behavior of 1100 aluminum in the strain rate range $10^{-5}$ $10^{4} \mathrm{~s}^{-1}$. Int J Plast, 1992, 8: 397-424

17 Khan A S, Liang R. Behaviors of three BCC metal over a wide range of strain rates and temperatures: Experiments and modeling. Int $\mathbf{J}$ Plast, 1999, 15: 1089-1109

18 Khan A S, Sung Suh Y, Kazmi R. Quasi-static and dynamic loading responses and constitutive modeling of titanium alloys. Int J Plast, 2004, 20: 2233-2248

19 Beyerlein I, Tomé C. A dislocation-based constitutive law for pure $\mathrm{Zr}$ including temperature effects. Int J Plast, 2008, 24: 867-895

20 Stainier L, Cuitiño A M, Ortiz M. A micromechanical model of hardening, rate sensitivity and thermal softening in bcc single crystals. J Mech Phys Solids, 2002, 50: 1511-1545

21 Johnson G R, Cook W H. A constitutive model and data for metals subjected to large strains, high strain rates and high temperatures. In: The Proceedings of the 7th International Symposium on Ballistics. Hague: Netherlands International Ballistics Committee, 1983

22 Zerilli F J, Armstrong R W. Dislocation-mechanics-based constitutive relations for material dynamics calculations. J Appl Phys, 1987, 61: 1816-1825

23 Baig M, Khan A S, Choi S H, et al. Shear and multiaxial responses of oxygen free high conductivity (OFHC) copper over wide range of strain-rates and temperatures and constitutive modeling. Int J Plast, 2013, 40: 65-80

24 Liang R, Khan A S. A critical review of experimental results and constitutive models for BCC and FCC metals over a wide range of strain rates and temperatures. Int J Plast, 1999, 15: 963-980

25 Khan A S, Kazmi R, Farrokh B. Multiaxial and non-proportional loading responses, anisotropy and modeling of Ti-6Al-4V titanium alloy over wide ranges of strain rates and temperatures. Int J Plast, 2007, 23: 931-950

26 Khan A S, Yu S. Deformation induced anisotropic responses of Ti-6Al-4V alloy. Part I: Experiments. Int J Plast, 2012, 38: 1-13

27 Khan A S, Yu S, Liu H. Deformation induced anisotropic responses of Ti-6Al-4V alloy Part II: A strain rate and temperature dependent anisotropic yield criterion. Int J Plast, 2012, 38: 14-26

28 Tang Q, Wang T, Shang B, et al. Thermodynamic properties and constitutive relations of crystals at finite temperature. Sci China-Phys Mech Astron, 2012, 55: 918-926

29 Tang Q H, Wang T C. Lattice wave theory of molecular dynamics (in Chinese). Sci Sin-Phys Mech Astron, 2011, 41: 214-220

30 Zbib H M, Diaz de la Rubia T. A multiscale model of plasticity. Int J
Plast, 2002, 18: 1133-1163

31 Roters F, Eisenlohr P, Hantcherli L, et al. Overview of constitutive laws, kinematics, homogenization and multiscale methods in crystal plasticity finite-element modeling: Theory, experiments, applications. Acta Mater, 2010, 58: 1152-1211

32 Kim J H, Kim D, Lee Y S, et al. A temperature-dependent elastoplastic constitutive model for magnesium alloy AZ31 sheets. Int $\mathbf{J}$ Plast, 2013, 50: 66-93

33 Li Z C, Jia H S, Ma H A, et al. FEM analysis on the effect of cobalt content on thermal residual stress in polycrystalline diamond compact (PDC). Sci China-Phys Mech Astron, 2012, 55: 639-643

34 Agarwal S, Briant C L, Krajewski P E, et al. Experimental validation of two-dimensional finite element method for simulating constitutive response of polycrystals during high temperature plastic deformation. J Mater Eng Perform, 2007, 16: 170-178

35 Ma A, Roters F. A constitutive model for fcc single crystals based on dislocation densities and its application to uniaxial compression of aluminium single crystals. Acta Mater, 2004, 52: 3603-3612

36 Zamiri A, Bieler T, Pourboghrat F. Anisotropic crystal plasticity finite element modeling of the effect of crystal orientation and solder joint geometry on deformation after temperature change. J Electron Mater, 2009, 38: 231-240

37 Asaro R J. Crystal plasticity. J Appl Mech, 1983, 50: 921-934

38 Liu X L, Tang Q H, Wang T C. A continuum thermal stress theory for crystals based on interatomic potentials. Sci China-Phys Mech Astron, 2014, 57: 1-10

39 Nix F C, MacNair D. The thermal expansion of pure metals: Copper, gold, aluminum, nickel, and iron. Phys Rev, 1941, 60: 597-605

40 Schmid E, Boas W. Plasticity of Crystals. London: F.A. Hughes \& Co. Limited, 1950

41 Suzuki T, Kamimura Y, Kirchner H. Plastic homology of bcc metals. Philos Mag A, 1999, 79: 1629-1642

42 Noguchi O, Oya Y, Suzuki T. The effect of nonstoichiometry on the positive temperature dependence of strength of $\mathrm{Ni}_{3} \mathrm{AI}$ and $\mathrm{Ni}_{3} \mathrm{Ga}$. Metall Trans. A, 1981, 12: 1647-1653

43 Suzuki T, Mishima Y, Miura S. Plastic behaviour in $\mathrm{Ni}_{3}(\mathrm{Al}, \mathrm{X})$ single crystal-temperature, strain-rate, orientation and composition. ISIJ Int, 1989, 29: 1-23

44 Suzuki T, Oya Y, Wee D M. Transition from positive to negative temperature dependence of the strength in $\mathrm{Ni}_{3} \mathrm{Ge}-\mathrm{Fe}_{3} \mathrm{Ge}$ solid solution. Acta Metall, 1980, 28: 301-310

45 Wee D M, Noguchi O, Oya Y, et al. New L12 ordered alloys having the positive temperature dependence of strength. Trans JIM, 1980, 21: 237

46 Howe S, Liebmann B, Lücke K. High temperature deformation of aluminum single crystals. Acta Metall, 1961, 9: 625-631

47 Gottstein G, Kocks U F. Dynamic recrystallization and dynamic recovery in $\langle 111\rangle$ single crystals of nickel and copper. Acta Metall, 1983, 31: 175-188

48 Anongba P, Bonneville J, Martin J. Hardening stages of [112] copper single crystals at intermediate and high temperatures-I. Mechanical behaviour. Acta Metall Mater, 1993, 41: 2897-2906

49 Stipp M, Stünitz H, Heilbronner R, et al. Dynamic recrystallization of quartz: Correlation between natural and experimental conditions. Geol Soci London Spec Pub, 2002, 200: 171-190

50 Mei J, Davenport J. Free-energy calculations and the melting point of Al. Phys Rev B, 1992, 46: 21-25

51 Adams M, Cottrell A. Effect of temperature on the flow stress of work-hardened copper crystals. Philos Mag, 1955, 46: 1187-1193

52 Takeuchi S, Kuramoto E. Temperature and orientation dependence of the yield stress in $\mathrm{Ni}\left\{\mathrm{in}_{3}\right\}$ Ga single crystals. Acta Metall, 1973, 21: 415-425

53 Lee M G, Lim H, Adams B L, et al. A dislocation density-based single crystal constitutive equation. Int J Plast, 2010, 26: 925-938

54 Izadbakhsh A, Inal K, Mishra R K, et al. New crystal plasticity constitutive model for large strain deformation in single crystals of magnesium. Comp Mater Sci, 2011, 50: 2185-2202 